\title{
MAXIMAL COHEN-MACAULAY MODULES AND THE QUASIHOMOGENEITY OF ISOLATED COHEN-MACAULAY SINGULARITIES
}

\author{
ALEX MARTSINKOVSKY \\ (Communicated by Maurice Auslander)
}

\begin{abstract}
We conjecture that a complete isolated Cohen-Macaulay singularity of dimension $\geq 2$ is graded if and only if sufficiently high syzygy modules of the residue field and of the transpose of the module of Kähler differentials are isomorphic. The "only if" part of the conjecture is proved for hypersurface singularities.
\end{abstract}

The purpose of this paper is to give module-theoretic conditions guaranteeing the quasihomogeneity of an isolated Cohen-Macaulay singularity. From a broader perspective, the existence of such conditions demonstrates, once again, the close ties between "representation" theory and "structure" theory. Our choice of maximal Cohen-Macaulay (or MCM, for short) modules ("representations") is not accidental. It is this category that has already proven itself to be closely connected with the geometry of the underlying ring (see introduction to [M1] for a brief description of the related results). A more conceptual explanation for our choice may possibly come from the theory of MCM approximations of Auslander and Buchweitz [AB].

The aforementioned conditions, besides being instructive and interesting in their own right, may also have a utilitarian purpose related to the classification problem of CM rings with only finitely many indecomposable MCM modules (nowadays called $\mathrm{CM}$ rings of finite (M)CM type). At the present moment all known examples of finite $\mathrm{CM}$ type are quasihomogeneous [AR]. Moreover, there can be no examples other than those known among homogeneous singularities $[\mathrm{EH}]$.

In $\S 0$ we put together a few basic facts about matrix factorizations and $\mathrm{MCM}$ approximation. Section 1 deals with the quasihomogeneity of isolated hypersurface singularities. We show that such a singularity is quasihomogeneous if and only if the MCM approximation of the corresponding moduli algebra has no

Received by the editors December 7, 1989.

1980 Mathematics Subject Classification (1985 Revision). Primary 13C99, 13H10, 13J10; Secondary $14 \mathrm{~B} 05$.

Key words and phrases. Cohen-Macaulay, quasihomogeneity, graded, isolated singularity, Kähler differentials, transpose, hypersurface, maximal Cohen-Macaulay approximations, moduli algebra. 
free summands. In $\S 2$ we formulate an extended (to arbitrary positive dimension $\geq 2$ ) version of author's Conjecture 2.4 from [M2] which characterizes the quasihomogeneity through a relation between the syzygetic behavior of the transpose of the module of Kähler differentials and the MCM approximation of either the maximal ideal or (depending on the parity of the dimension) the residue field. We then investigate this relation for quasihomogeneous hypersurface singularities.

Our rings are analytic $k$-algebras, understood here as quotients of formal power series rings over a field $k$. An isolated singularity is then defined through the Jacobian criterion, where the module of Kähler differentials is the universally finite module of differentials.

An analytic $k$-algebra $(R, \underline{m})$ is called graded if there exist a system of generators $x_{1}, \ldots, x_{n}$ of the maximal ideal, positive integers $d_{1}, \ldots, d_{n}$, and a $k$-derivation $\delta: R \rightarrow R$ such that $\delta x_{i}=d_{i} x_{i}, i=1, \ldots, n$. The elements of the eigenspace $V_{l}$ are said to be homogeneous of degree $l$. (Unlike the case of affine algebras, the direct sum of $V_{l}$ does not span the whole ring $R$.) The words "graded" and "quasihomogeneous" are synonymous. If $d_{i}=1, i=1, \ldots, n$, the singularity is called homogeneous.

\section{BACKGROUND MATERIAL}

0.1 . Matrix factorizations (see $[\mathrm{E}]$ for details). Let $(A, \underline{m})$ be a regular local ring and $x \in \underline{m}$. A matrix factorization of $x$ is an ordered pair of homomorphisms $(\varphi: F \rightarrow G, \psi: G \rightarrow F)$ of free $A$-modules $F$ and $G$ such that $\varphi \psi=x \cdot \operatorname{Id}_{G}$ and $\psi \varphi=x \cdot \operatorname{Id}_{F}$. It turns out that $\operatorname{rk} F=\operatorname{rk} G$. Two matrix factorizations $(\varphi: F \rightarrow G, \psi: G \rightarrow F)$ and $\left(\varphi^{\prime}: F^{\prime} \rightarrow G^{\prime}, \psi^{\prime}: G^{\prime} \rightarrow F^{\prime}\right)$ are called equivalent if there exist isomorphisms $\alpha: F \rightarrow F^{\prime}$ and $\beta: G \rightarrow G^{\prime}$ such that $\varphi^{\prime} \alpha=\beta \varphi$ and $\alpha \psi=\psi^{\prime} \beta$. The factorization $(\varphi, \psi)$ is called reduced if $\operatorname{Im}(\varphi) \subset \underline{m} G$ and $\operatorname{Im}(\psi) \subset \underline{m} F$. If $B:=A /(x)$ and overbar denotes reduction modulo $x$ then the periodic complex $\mathbb{F}(\varphi, \psi): \cdots \bar{F} \stackrel{\bar{\varphi}}{\longrightarrow} \bar{G} \stackrel{\bar{\psi}}{\longrightarrow} \bar{F} \stackrel{\bar{\varphi}}{\longrightarrow} \bar{G}$ is a $B$-free resolution of the MCM $B$-module Coker $\varphi$. Moreover the associations $(\varphi, \psi) \rightarrow \mathbb{F}(\varphi, \psi)$ and $(\varphi, \psi) \rightarrow \operatorname{Coker} \varphi$ induce bijections between the sets of

(1) equivalence classes of reduced matrix factorizations of $x$ over $A$,

(2) isomorphism classes of nontrivial 2-periodic minimal free resolutions over $B$, and

(3) MCM $B$-modules without free summands.

0.2. MCM approximations. The results described here are due to Auslander and Buchweitz and can be found in the lecture notes [A] (unfortunately, not in the printed form yet) and $[\mathrm{AB}]$. Throughout this subsection $R$ is a complete local CM ring.

Definition 1. Let $X$ be an $R$-module. A map $p: M \rightarrow X$ is called an MCM approximation of $X$ if $M$ is an MCM module and for any MCM module $N$ 
and any map $f: N \rightarrow X$ there exists a map $g: N \rightarrow M$ such that $f=p g$.

An MCM approximation $p: M \rightarrow X$ is called minimal if no direct summand $M^{\prime}$ of $M$ is part of an MCM approximation $p^{\prime}: M^{\prime} \rightarrow X$. Taking $N \cong R$ we see that $p$ is necessarily surjective. Minimal approximations always exist and are uniquely determined up to (nonnatural) isomorphisms. Henceforth we will only consider minimal MCM approximations.

This same concept can also be described by the equivalent

Definition 2. Let $X$ be an $R$-module. An MCM approximation of $X$ is a short exact sequence $0 \rightarrow V \rightarrow M \stackrel{p}{\longrightarrow} X \rightarrow 0$ of $R$-modules where $M$ is MCM and $V$ has finite injective dimension.

This approximation is called minimal if $V$ and $M$ have no common direct summands.

Suppose now that $R$ is a hypersurface ring of dimension $d-1$ and $X$ an $R$-module. By [E] a minimal resolution of $X$ becomes 2-periodic at the $d$ th step. We thus have the diagram

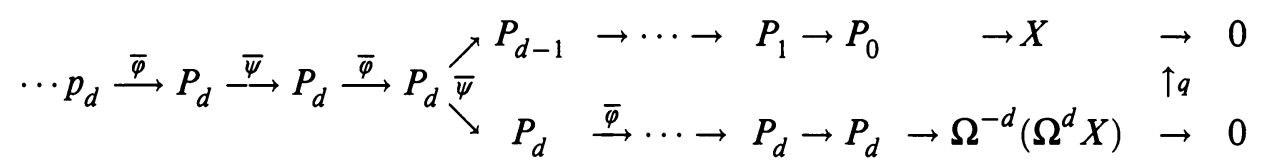

where $\Omega^{n}$ stands for the $n$th syzygy module and $q$ is an iterated lifting of the identity map of $\Omega^{d} X$. It turns out that, in a slightly abused language, $\Omega^{-d}\left(\Omega^{d} X\right)$ is the nonprojective part of the MCM approximation of $X$, and if $q^{\prime}: Q \rightarrow X / \operatorname{Im} q$ is a projective cover, then, upon identifying $q^{\prime}$ with its lifting to $X$, the map $q \amalg q^{\prime}: \Omega^{-d}\left(\Omega^{d} X\right) \amalg Q \rightarrow X$ is a minimal MCM approximation of $X$. Notice that if $Q$ is a nonzero module then $q$ is not surjective.

We begin with the results originally proved by the author (see [M1, §10]) for $n=3$.

Proposition 1.1. Let $P:=k\left[\left|X_{1}, \ldots, X_{n}\right|\right]$ be the formal power series ring in $n$ variables over a field $k$ of characteristic $0, f \in \underline{m}_{p}:=\left(X_{1}, \ldots, X_{n}\right)$ a formal power series whose Jacobian ideal $j(f)$ is $\underline{m}_{P}$-primary and $R:=P /(f)$. Then the following are equivalent.

(1) The moduli algebra $R / \overline{j(f)}$ (the overbar denotes the image in $R$ ) is Gorenstein.

(2) $f \in j(f)$.

(3) $f \in \underline{m}_{P} j(f)$.

(4) there exist an MCM module $N$ without free summands and a surjection $\alpha: N \rightarrow R / \overline{j(f)}$.

(5) The MCM approximation of the moduli algebra $R / \overline{j(f)}$ has no free summands. 
Proof. (1) $\Leftrightarrow(2)$ is known. To prove the nontrivial implication (1) $\Rightarrow(2)$ we first remark that by $[\mathrm{K}]$ the Gorenstein ring $R / \overline{j(f)} \cong P /(f, j(f))$ cannot be an almost complete intersection. Thus either $f \in j(f)$ and we are done, or one of the partials of $f$ is a linear combination of $f$ and other partials: $\partial f / \partial x_{i}=\sum_{j \neq i} a_{j} \partial f / \partial x_{j}+b f, b, a_{j} \in P$. This relation gives rise to the $k$-derivation $\delta:=\partial / \partial x_{i}-\sum_{j \neq i} a_{j} \partial / \partial x_{j}$ of $P$ which preserves the ideal $(f)$ and thus induces a $k$-derivation $\bar{\delta}$ of $R$ which has a unit in its image (since $\left.\bar{\delta}\left(\bar{X}_{i}\right)=1\right)$. This contradicts the result of Zariski asserting that the images of derivations of isolated singularities over a field of characteristic 0 are contained in the maximal ideal (see [Te, p. 586]).

(2) $\Leftrightarrow(3)$ is obvious since if $f \in j(f)-\underline{m}_{p} j(f)$ then one of the partials is a linear combination of $f$ and other partials, the case just ruled out.

$(4) \Leftrightarrow(5)$ is true for an arbitrary $R$-module in place of $R / \overline{j(f)}$. Suppose that $\beta: M \amalg Q \rightarrow R / \overline{j(f)}$ is an MCM approximation with $Q$ a nonzero projective. By the universal property of approximations the surjection $\alpha: N \rightarrow R / \overline{j(f)}$ factors through $\beta: \alpha=\beta \gamma$ for some $\gamma: N \rightarrow M \amalg Q$. Since $\alpha$ is surjective and $\beta \mid M$ is not (see the end of $\S 0.2$ ), the image of $\gamma$ must contain an element from $Q-\underline{m}_{R} Q$. But then $N$ has a free summand, a contradiction.

$(3) \Leftrightarrow(4)$ holds for an arbitrary ideal in place of $j(f)$ as was shown by Ding in his forthcoming thesis.

Corollary. Suppose that, in addition to the assumptions of Proposition 1, the field $k$ is algebraically closed. Then $R$ is graded if and only if the moduli algebra $R / \overline{j(f)}$ is a homomorphic image of an MCM module without free summands.

Proof. Under our assumptions, the fundamental result of K. Saito ([Sa], [SchW, Satz 5.7]) says that $R$ is graded if and only if $f \in j(f)$.

\section{A GENERAL CONJECTURE}

The results of the previous section proven for isolated hypersurface singularities suggest a possibility that there is a module-theoretic criterion for the gradedness of an arbitrary analytic $k$-algebra with an isolated singularity. In fact, in [M2] we conjectured that a two-dimensional integrally closed analytic $k$-algebra $R$ is graded if and only if the Auslander module of $R$ is isomorphic to the module of Zariski differentials of $R$, and also proved the conjecture in certain cases. Further supporting evidence was provided by Behnke [B]. The purpose of this section is to state an analog of the aforementioned conjecture for an arbitrary positive dimension and then consider the hypersurface case.

Conjecture 2.1. Let $(R, \underline{m})$ be a complete isolated $C M$ singularity of dimension $d \geq 2$ over a field $k$ of characteristic 0 . Then $R$ is graded if and only if $\left(\Omega^{d}\left(\operatorname{Tr}\left(D_{k}(R)\right)\right)^{*}\right.$ is isomorphic to the MCM approximation of the maximal ideal when $d$ is even and to the MCM approximation of the residue field when $d$ is odd. Here "*" stands for $\operatorname{Hom}_{R}(-, R), \Omega^{d}(-)$ is the dth syzygy module of "-," $\operatorname{Tr}$ is the transpose of "-" (i.e., the cokernel of the transpose of a minimal 
presentation matrix of "-") and $D_{k}(R)$ is the module of Kähler differentials of $R$ over $k$.

Let $d=2$. Dualizing a minimal presentation $P_{1} \rightarrow P_{0} \rightarrow D_{k}(R)$ we have the exact sequence

$$
0 \rightarrow D_{k}(R)^{*} \rightarrow P_{0}^{*} \stackrel{\Delta^{*}}{\longrightarrow} P_{1}^{*} \rightarrow \operatorname{Tr}\left(D_{k}(R)\right) \rightarrow 0
$$

which shows that $\Omega^{2}\left(\operatorname{Tr}\left(D_{k}(R)\right)\right)^{*} \cong D_{k}(R)^{* *}$. Since the MCM approximation of the maximal ideal is the Auslander module of $R$, when $d=2$ Conjecture 2.1 becomes Conjecture 2.4 of [M2].

Our goal is to prove the "only if" part of the conjecture when $R$ is a hypersurface and $k$ is algebraically closed.

Proposition 2.2. Let $R=P /(f)$ where $P=k\left[\left|X_{1}, \ldots, X_{n}\right|\right]$ is a formal power series ring over an algebraically closed field $k$ of characteristic 0 , and $f \in \underline{m}_{P}:=$ $\left(X_{1}, \ldots, X_{n}\right)$ a formal power series with $\underline{m}_{P}$-primary Jacobian ideal $j(f)$. If $R$ is graded then $\Omega^{n-1}(R / \overline{j(f)})^{*}$ is isomorphic to the MCM approximation of the maximal ideal $\underline{m}_{R}$ of $R$ when $n-1$ is even, and to the MCM approximation of $k$ when $n-1$ is odd.

Proof. By Satz 5.7 of [SchW] there exists a grading of $P$ with respect to which $f$ becomes a homogeneous polynomial. Thus without loss of generality we may assume that each $X_{i}$ is homogeneous of degree $d_{i}, i=1, \ldots, n$, and $f$ is a homogeneous polynomial of degree $d$ (in the chosen grading). Applying the Euler derivation we have

$$
f=\sum_{i=1}^{n} y_{i} \partial f / \partial X_{i},
$$

where $y_{i}=d_{i} X_{i} / d \neq 0$. Notice that the elements $y_{i}, i=1, \ldots, n$, generate the maximal ideal of $P$. Therefore to obtain the MCM approximation of the residue field (and the maximal ideal) of $R$ it suffices to construct a projective resolution over $R$ of $R /\left(\bar{y}_{1}, \ldots, \bar{y}_{n}\right)$. This can be accomplished with the aid of the Tate resolution (see [Ta, p. 20, Theorem 4]). But the partials of $f$, by hypothesis, also form a system of parameters and the same gadget gives a projective resolution of the moduli algebra $R /\left(\overline{\partial f / \partial X_{i}}, \ldots, \overline{\partial f / \partial X_{n}}\right)$. Thus our proposition is a statement about the properties of the Tate resolution. More precisely, in the notation of [Ta] the $R$-algebra $Y:=R\left\langle T_{1}, \ldots, T_{n}, S\right\rangle$ with each $T_{i}$ of degree 1 and $S$ of degree 2, and with differential $d$ defined by

$$
d T_{i}:=\bar{y}_{i}, \quad d S:=\sum_{i=1}^{n}\left(\overline{\partial f / \partial X_{i}}\right) T_{i}
$$

yields a projective resolution of the $R$-module $R /\left(\bar{y}_{1}, \ldots, \bar{y}_{u}\right)=R / \underline{m}_{R}$. On the other hand if we define differential $d^{\prime}$ by

$$
d^{\prime} T_{i}:=\overline{\partial f / \partial X_{i}}, \quad d^{\prime} S:=\sum_{i=1}^{n} \bar{y}_{i} T_{i}
$$


we obtain a projective resolution of the $R$-module $R / \overline{j(f)}$. Notice that the elements $y_{i}$ and $\partial f / \partial X_{i}$ can be viewed in (1) as either parameters or their coefficients. Utilizing this we introduce a symbolic involution $\mathscr{I}$ acting on those elements by

$$
\mathscr{I}\left(\partial f / \partial x_{i}\right):=y_{i}, \quad \mathscr{I}\left(y_{i}\right):=\partial f / \partial X_{i}, \quad i=1, \ldots, n .
$$

We will also consider the obvious action of $\mathscr{I}$ on their images in $R$.

Let $d_{k}$ (resp. $d_{k}^{\prime}$ ) be the degree $k$ homogeneous part $Y_{k+1} \rightarrow Y_{k}$ of $d$ (resp. $\left.d^{\prime}\right)$. Since the corresponding matrices have $\bar{y}_{i}$ and $\overline{\partial f / \partial X_{i}}$ as their nonzero entries, we can lift those matrices to $P$ in the obvious way, where, without the danger of confusion, we denote them by the same letters. Involution $\mathscr{I}$ acts on their entries and we have the obvious identities

$$
\mathscr{I}\left(d_{k}\right)=d_{k}^{\prime}, \quad \mathscr{I}\left(d_{k}^{\prime}\right)=d_{k} \text {. }
$$

In our new notation, $\left(\Omega^{n-1}(R / \overline{j(f)})\right)^{*} \cong\left(\operatorname{Coker} d_{n-1}^{\prime}\right)^{*}$. On the other hand, if $n$ is odd the MCM approximation of the maximal ideal is isomorphic to Coker $d_{n}$, which follows from the construction of approximations via negative syzygies; and if $n$ is even the MCM approximation of the residue field is also isomorphic to Coker $d_{n}$. Thus we want to show that Coker $d_{n} \cong\left(\operatorname{Coker} d_{n-1}^{\prime}\right)^{*}$, or, utilizing (4),

$$
\text { Coker } d_{n} \cong\left(\operatorname{Coker} \mathcal{F}\left(d_{n-1}\right)\right)^{*} .
$$

Since Coker $d_{n-1}^{\prime}$ is MCM, we have $\left(\operatorname{Coker} \mathscr{I}\left(d_{n-1}\right)\right)^{*} \cong\left(\operatorname{Coker}\left(\mathscr{I}\left(d_{n-1}\right)\right)^{t}\right)$ where $t$ denotes the transpose of a matrix. Thus we want to show that matrices $d_{n}$ and $\mathscr{I}\left(d_{n-1}\right)^{t}$ have isomorphic cokernels.

Since rk $Y_{n-1}=\operatorname{rk} Y_{n}=\cdots=2^{n-1}$, Coker $d_{n-1}$ has no free summands and thus comes from a reduced matrix factorization, say, $\left(d_{n-1}, \Phi\right)$, where the matrices are viewed as matrices with entries in $P$ and the $R$-modules Coker $\Phi$ and Coker $d_{n}$ are isomorphic. Since $\Phi$ is uniquely determined by $d_{n-1}$, it suffices to show that $\left(d_{n-1}, \mathcal{I}\left(d_{n-1}\right)^{t}\right)$ is also a matrix factorization. In fact, it suffices to show that $\mathscr{I}\left(d_{n-1}\right)^{t} \cdot d_{n-1}$ is the scalar matrix with $f$ on the diagonal (the dot denotes the usual matrix product). In other words, for any two column vectors $v_{i}$ and $v_{j}$ of $d_{n-1}$ we must have $\mathscr{I}\left(v_{i}\right)^{t} \cdot v_{j}=\delta_{i j} f$.

To prove this it is convenient to introduce new notation. Let $I$ denote an increasing map from the set $[l]$ of integers $\{0,1, \ldots, l\}$ to the set $[n]=$ $\{0,1, \ldots, n\}$ (an "l-subsimplex" of the " $n$-simplex"). Choosing the $R$-basis $T_{i_{1}}, T_{i_{2}}, \ldots, T_{i_{l}} S^{(p)}, l+2 p=m$ for $Y_{m}, m=1,2, \ldots$, we can use our new notation as shorthand: $T_{I} S^{(p)}$. Let $I_{j}$ denote the map $[l-1] \rightarrow[n]$ defined by

$$
I_{j}(q)=\left\{\begin{array}{ll}
I(q) & \text { if } q<j \\
I(q+1) & \text { if } q \geq j,
\end{array} \quad j=1, \ldots, l-1\right.
$$

(this is the " $j$ th submaximal face of $I$ "), and let CI denote the complement $[n] \backslash \operatorname{Im}(I)$ of the image of $I$ in $[n]$ (the "vertices of $[n]$ not in $I$ "). A trivial 
computation shows that

$$
\begin{aligned}
d_{m-1}\left(T_{I} S^{(p)}\right) & =\sum_{j \in[l]}(-1)^{j-1} \bar{y}_{I(j)} T_{I_{j}} S^{(p)}+(-1)^{l} T_{I}\left(\sum_{k \in[n]}\left(\overline{\partial f / \partial x_{k}}\right) T_{k} S^{(p-1)}\right) \\
& =\sum_{j \in[l]}(-1)^{j-1} \bar{y}_{I(j)} T_{I_{j}} S^{(p)}+(-1)^{l} \sum_{k \in C I}\left(\overline{\partial f / \partial x_{k}}\right) T_{I} T_{k} S^{(p-1)} .
\end{aligned}
$$

Now it is immediate that if $v$ is the column-vector of $d_{m-1}$ (with entries in $P$ ) corresponding to $d_{m-1}\left(T_{I} S^{(p)}\right)$ with $p>0$ or with $p=0$ and $l=n$, then $\mathscr{I}(v)^{t} \cdot v=\sum_{i \in[n]} y_{i}\left(\partial f / \partial x_{i}\right)=f$ (by (1)). In particular

$$
\mathscr{I}(v)^{t} \cdot v=f \text { if } m=n .
$$

Now we choose two different basis elements $T_{I} S^{(p)}$ and $T_{J} S^{(q)}$ from $Y_{n}$, where $I$ is an increasing map $[l] \rightarrow[n]$ and $J$ is an increasing map $\left[l^{\prime}\right] \rightarrow[n]$ with $n=l+2 p=l^{\prime}+2 q$. Then as before,

$$
\begin{aligned}
& d_{n-1}\left(T_{I} S^{(p)}\right)=\sum_{j \in[l]}(-1)^{j-1} \bar{y}_{I(j)} T_{I_{j}} S^{(p)}+(-1)^{l} \sum_{k \in C I}\left(\overline{\partial f / \partial x_{k}}\right) T_{I} T_{k} S^{(p-1)}, \\
& d_{n-1}\left(T_{J} S^{(q)}\right)=\sum_{j \in\left[l^{\prime}\right]}(-1)^{i-1} \bar{y}_{J(i)} T_{J_{i}} S^{(q)}+(-1)^{l^{\prime}} \sum_{h \in C J}\left(\overline{\partial f / \partial x_{h}}\right) T_{J} T_{h} S^{(q-1)} .
\end{aligned}
$$

If the corresponding column-vectors $v_{I}$ and $v_{J}$ are such that $\mathscr{I}\left(v_{J}\right)^{t} \cdot v_{I}=0$ we have nothing to prove. Otherwise we can have the four cases:

(1) $\exists j \in[l]$ and $\exists i \in\left[l^{\prime}\right]$ such that $T_{I_{j}} S^{(p)}=T_{J_{i}} S^{(q)}$;

(2) $\exists j \in[l]$ and $\exists h \in C J$ such that $T_{I_{j}} S^{(p)}=( \pm) T_{J} T_{h} S^{(q-1)}$;

(3) $\exists i \in\left[l^{\prime}\right]$ and $\exists k \in C I$ such that $T_{J_{i}} S^{(q)}=( \pm) T_{I} T_{k} S^{(p-1)}$;

(4) $\exists k \in C J$ and $\exists h \in C J$ such that $T_{I} T_{k} S^{(p-1)}=( \pm) T_{J} T_{h} S^{(q-1)}$.

Case 1. It is clear that $I_{j}=J_{i}$, hence $l=l^{\prime}$ and $p=q$. The corresponding contribution to the product is equal to $(-1)^{i+j}\left(\partial f / \partial x_{J(i)}\right) y_{I(j)}$. Since the basis elements $T_{I} S^{(p)}$ and $T_{J} S^{(q)}$ are different we may assume, without loss of generality, that $J(i)<I(j)$ (and, therefore, $i \leq j)$. Symbolically the sets $I([l])$ and $J\left(\left[l^{\prime}\right]\right)$ look as follows:

$I([l])$

$J\left(\left[l^{\prime}\right]\right) \quad \cdot \quad \cdot \quad \underset{J(i)}{\circ}$

where the black dots indicate the elements in the image of $I_{j}=J_{i}$. It is obvious that $I([l]) \cup J(i)=J\left(\left[l^{\prime}\right]\right) \cup I(j)$. We also notice that $p>0$, otherwise $n=l=l^{\prime}$ and $I=J$, contrary to the assumption. Therefore $T_{I} T_{J(i)} S^{(p-1)}=$ $( \pm) T_{J} T_{I(j)} S^{(p-1)}$ is a basis element in $Y_{n-1}$. If we now define an increasing 
map $H:[l+1] \rightarrow[n]$ by

$$
H(t)= \begin{cases}I(t)=J(t), & \text { if } t<i, \\ J(i), & \text { if } t=i, \\ I(t-1)=J(t), & \text { if } i<t \leq j, \\ I(j), & \text { if } t=j+1, \\ I(t-1)=J(t-1), & \text { if } t>j+1,\end{cases}
$$

then $T_{I} T_{J(i)} S^{(p-1)}=(-1)^{l-i+1} T_{H} S^{(p-1)}$ and $T_{J} T_{I(j)} S^{(p-1)}=(-1)^{l-j} T_{H} S^{(p-1)}$ (remember $J(i)<I(j) !)$. The contributions to $\mathscr{I}\left(v_{J}\right)^{t} \cdot v_{I}$ from the components corresponding to $T_{H} S^{(p-1)}$ will then be $(-1)^{1-i-j} y_{I(j)}\left(\partial f / \partial x_{J(i)}\right)$ which cancels the previously computed product. Thus there can be no nonzero contribution to $\mathscr{I}\left(v_{J}\right)^{t} \cdot v_{I}$ in Case 1 .

Case 2. Now $p=q-1$ and $l^{\prime}=l-2$, and $\operatorname{Im}\left(I_{j}\right)=J([l-2]) \cup\{h\}$. In particular, there exists $j^{\prime} \in[l]$ such that $h=I\left(j^{\prime}\right)$. First we assume that $j^{\prime}<j$. Symbolically we are in the following situation:

$I([l])$

$J([l-2])$

$$
\stackrel{\circ}{h=I\left(j^{\prime}\right)} \quad \cdot \quad \stackrel{\circ}{h^{\prime}=I(j)}
$$

where the black dots indicate the elements of $J([l-2]) \subset I([l])$. The product of the components corresponding to the basis element

$$
T_{I_{j}} S^{(p)}=( \pm) T_{J} T_{h} S^{(q-1)} \text { is equal to }(-1)^{j-1} y_{I(j)}\left((-1)^{l-2+j^{\prime}-1} y_{I\left(j^{\prime}\right)}\right) .
$$

But we also have $T_{I_{j}} S^{(p)}=( \pm) T_{J} T_{h^{\prime}} S^{(q-1)}$, where $h^{\prime}:=I(j)$, and the corresponding contribution to $\mathscr{I}\left(v_{J}\right)^{t} \cdot v_{I}$ is $(-1)^{j^{\prime}-1} Y_{I\left(j^{\prime}\right)}\left((-1)^{l-2+j-2}\right)$ (since $j^{\prime}<j$ !), which cancels the previously computed product.

If $j<j^{\prime}$ the argument is identical to the one just given, and the case $j=j^{\prime}$ can never be realized since $J([l-2]) \cup I\left(j^{\prime}\right)=\operatorname{Im} I_{j}$ and $I(j) \notin \operatorname{Im} I_{j}$.

Case 3. This case is completely analogous to Case 2 . We should remark that examining the relations between $p$ and $q$ we see that no two of the three considered cases can happen simultaneously: we either have $p=q$ or $p=q-1$ or $q=p-1$. This remark actually legitimizes our argument. We now proceed to

Case 4. Here $I([l]) \cup k=J\left(\left[l^{\prime}\right]\right) \cup h$ and $l=l^{\prime}$. If $k=h$ then $I=J$, contrary to the assumption. If $k \neq h$ then $k=J(i)$ for some $i \in[l]$ and $h=I(j)$ for some $j \in[l]$. Symbolically:

$$
I(j)=k
$$


where the black dots indicate the elements of $I([l]) \cap J([l])$. But then $I_{j}=J_{i}$, which is the possibility considered in Case 1 . In fact $I_{j}=J_{i}$ if and only if $T_{I} T_{k}=( \pm) T_{J} T_{h}$, where $k=J(i)$ and $h=I(j)$. Thus Case 4 is exactly Case 1.

We now see that $\mathscr{F}\left(v_{J}\right)^{t} \cdot v_{I}=\delta_{I J} f$, and Proposition 2.2 is proven.

Remarks. (1) We have actually proven more than intended. Namely, if $v_{I}$ and $v_{J}$ are the column-vectors of $d_{m-1}$ corresponding to $d_{m-1}\left(T_{I} S^{(p)}\right)$ and $d_{m-1}\left(T_{J} S^{(q)}\right)$, where $I:[l] \rightarrow[n]$ and $J:\left[l^{\prime}\right] \rightarrow[n]$ are increasing maps and $m=l+2 p=l^{\prime}+2 q$, then:

(a) $\mathscr{I}\left(v_{J}\right)^{t} \cdot v_{I}=\delta_{I J} f$ if $p>0$ or if $p=0$ and $l=n(=m)$.

(b) If $I \neq J$ then $v_{I}$ and $v_{J}$ either have no common nonzero components or they have exactly two.

It is not difficult to show that similar assertions about the row-vectors of the differentials $d_{m}$ are true without any restrictions (in particular, for any rowvector $r$ we have $\left.r \cdot \mathscr{J}(r)^{t}=f\right)$.

Clearly the assertions of this remark are true for any system of parameters $y_{1}, \ldots, y_{n}$ of a regular local ring $(P, \underline{m})$ and any defining equation $f \in \underline{m}\left(y_{1}, \ldots, y_{n}\right)$.

(2) Conjecture 2.1 is now proven in the following cases:

$\operatorname{dim} 2$ : quotient singularities, graded Gorenstein rings, hypersurfaces $z^{n}+$ $f(x, y)$ ( $k$ is algebraically closed) (see [M2], as well as minimally elliptic singularities and rational singularities with reduced fundamental cycle (see [B]) (in the last two cases $k=\mathbb{C}$ ). In dimension 2 the "only if" part of the conjecture is always true if $k=\mathbb{C}(\mathrm{J}$. Wahl).

In every positive dimension the "only if" part is true for hypersurfaces $(k$ is algebraically closed).

\section{REFERENCES}

[A] M. Auslander, Cohen-Macaulay approximations, lecture notes of a course given at Brandeis University, Fall 1987.

[AB] M. Auslander and R.-O. Buchweitz, The homological theory of maximal Cohen-Macaulay approximations, preprint, Univ. of Toronto, 1988.

[AR] M. Auslander and I. Reiten, The Cohen-Macaulay type of Cohen-Macaulay rings, Adv. in Math. (to appear).

[B] K. Behnke, On Auslander modules of normal surface singularities, preprint, Matematisches Seminar Universität Hamburg, 1988.

[E] D. Eisenbud, Homological algebra on a complete intersection, Trans. Amer. Math. Soc. 260 (1980), 35-64.

[EH] D. Eisenbud and J. Herzog, The classification of homogeneous Cohen-Macaulay rings of finite representation type, Math. Ann. 280 (1988), 347-352.

[K] E. Kunz, Almost complete intersections are not Gorenstein rings, J. Algebra 28 (1974), 111 115. 
[M1] A. Martsinkovsky, Almost split sequences and Zariski differentials, Thesis, Brandeis Univ., 1987.

[M2] _ Almost split sequences and Zariski differentials, Trans. Amer. Math. Soc. (to appear).

[Sa] K. Saito, Quasihomogene isolierte Singularitäten von Hyperflächen, Invent. Math. 14 (1971), 123-142.

[SchW] G. Scheja and H. Wiebe, Über Derivationen von lokalen analytischen Algebren, Sympos. Math. XI (1973), 161-192.

[Ta] J. Tate, Homology of Noetherian rings and local rings, Illinois J. Math (1957), 14-27.

[Te] B. Teissier, The hunting of invariants in the geometry of discriminants, Proc. Nordic Summer School, Oslo, 1976.

Department of Mathematics, Northeastern University, Boston, Massachusetts 02115 